\title{
Short-time dynamics of polypeptides
}

Everaldo Arashiro, J. R. Drugowich de Felício, and Ulrich H. E. Hansmann

Citation: The Journal of Chemical Physics 126, 045107 (2007); doi: 10.1063/1.2430709

View online: https://doi.org/10.1063/1.2430709

View Table of Contents: http://aip.scitation.org/toc/jcp/126/4

Published by the American Institute of Physics

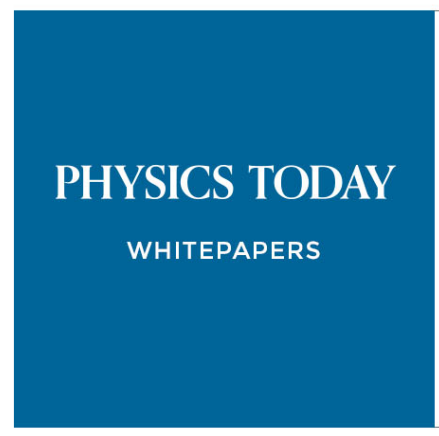




\title{
Short-time dynamics of polypeptides
}

\author{
Everaldo Arashiro a) \\ John v. Neumann Institute for Computing (NIC), Forschungszentrum Jülich, 52425 Jülich, Germany
}

J. R. Drugowich de Felício ${ }^{\text {b) }}$

Departamento de Física e Matemática, FFCLRP Universidade de São Paulo, Avenida Bandeirantes 3900, Ribeirão Preto, São Paulo CEP 14040-901, Brazil

Ulrich H. E. Hansmann ${ }^{\mathrm{c})}$

John v. Neumann Institute for Computing (NIC), Forschungszentrum Jülich, 52425 Jülich, Germany and Department of Physics, Michigan Technological University, Houghton, Michigan 49931-1291

(Received 24 October 2006; accepted 8 December 2006; published online 31 January 2007)

The authors study the short-time dynamics of helix-forming polypeptide chains using an all-atom representation of the molecules and an implicit solvation model to approximate the interaction with the surrounding solvent. The results confirm earlier observations that the helix-coil transition in proteins can be described by a set of critical exponents. The high statistics of the simulations allows the authors to determine the exponent values with increased precision and support universality of the helix-coil transition in homopolymers and (helical) proteins. () 2007 American Institute of Physics. [DOI: $10.1063 / 1.2430709$ ]

\section{INTRODUCTION}

While energy landscape theory and funnel concept ${ }^{1,2}$ are powerful tool for describing the general characteristics of folding, many details remain to be solved. One example is the nature of the transitions between different thermodynamic states that are observed during folding of proteins. Their nature and role in the folding process are active areas of research as many diseases are associated with spontaneous or induced misfolding of proteins. In principle, these transitions can be probed in silico. However, the energy landscape of proteins is characterized by a rough energy landscape with a huge number of local minima separated by high energy barriers. Considerable effort has been put into the development of sampling techniques that overcome the resulting slow convergence of computer experiments. ${ }^{3,4}$ In generalized-ensemble techniques ${ }^{5}$ such as parallel tempering, ${ }^{6}$ the broad-histogram method, ${ }^{7}$ or multicanonical sampling, ${ }^{8}$ simulations are performed in an artificial ensemble where the convergence to the equilibrium distribution is fast. Thermodynamic quantities are then calculated through reweighting to the canonical (i.e., physical) ensemble. In Ref. 9 we applied a complimentary approach to protein science that leaves the "physical" dynamics unchanged. The underlying idea is to extract information on certain equilibrium properties from the critical relaxation of regular canonical Monte Carlo simulations (i.e., a nonequilibrium behavior of the system).

Here, we revisit this approach and study with much improved statistics the helix-coil transition in simple polypeptides. Since it allows us to compare our results with earlier work, we study in the present paper the nonequilibrium evo-

\footnotetext{
${ }^{a)}$ Electronic mail: e.arashiro@fz-juelich.de

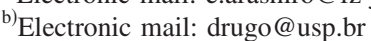

${ }^{c)}$ Electronic mail: hansmann@mtu.edu and u.hansmann@fz-juelich.de
}

lution process of $(\mathrm{Ala})_{N}$ chains $(N=10,20,30$, and 40) in short-time Monte Carlo (MC) simulations. Our investigation is later extended towards the 34-residue human parathyroid hormone $[\mathrm{PTH}(1-34)]$ fragment. We evaluate and compare for both systems the exponents that characterize the observed helix-coil transition.

\section{METHODS}

Janssen et al. ${ }^{10}$ and Huse ${ }^{11}$ have shown that at criticality universality and scaling are already present early in the evolution of systems. A system, characterized by an order parameter $m$, decays at a critical temperature $T=T_{c}$ from an initially ordered state $m_{0}=1$ (after a microscopic time $t_{\text {mic }}$ ) according to a power law ${ }^{12-14} m(t) \sim t^{-\beta / v z}$. This relation is obtained from the more general scaling form,

$$
m(t, \tau, N)=b^{-\beta / \nu} m\left(b^{-z} t, b^{1 / \nu} \tau, b^{-1} N\right),
$$

where $b$ is an arbitrary chain size scaling factor and $\tau$ is the reduced temperature, $\tau=\left(T-T_{c}\right) / T_{c}$. Differentiating $\ln m(t, \tau)$ with respect to the temperature $T$ at $T=T_{c}$,

$$
\left.\frac{\partial \ln m(t, \tau, L)}{\partial \tau}\right|_{\tau=0} \sim t^{1 / \nu z},
$$

leads to a scaling relation ${ }^{15}$ that allows one to extract the exponent $1 / \nu z$. Combining the two scaling relations allows one further to eliminate the dynamic exponent $z$ and to calculate the estimates of the critical exponent $\beta$. Additional information on the system can be obtained from the scaling ${ }^{16}$ of the second cumulant

$$
U_{2}(t, L)=\frac{m^{2}(t, L)}{(m(t, L))^{2}} \sim t^{d / z} .
$$

We use in the present paper Eq. (3) in combination with Eq. (2) to obtain estimates of the exponent $d \nu$. 
Another dynamic critical exponent was proposed by Majumdar et al. ${ }^{17}$ The authors studied global persistence probability $P(t)$ which is defined as

$$
P(t)=1-\sum_{t^{\prime}=1}^{t} \rho\left(t^{\prime}\right),
$$

where $\rho\left(t^{\prime}\right)$ is the fraction of the samples where the order parameter $m$ has changed its sign for the first time at the instant $t^{\prime}$. At criticality, $P(t)$ is expected to decay algebraically as

$$
P(t) \sim t^{-\theta_{g}},
$$

where $\theta_{g}$ is the global persistence exponent. The study of the persistence behavior has attracted enormous interest, playing an important role in the study of systems far from equilibrium. $^{18-24}$

The question arises whether the above scaling laws apply also to transitions in proteins and other biological molecules for that - as finite systems - the concept of a phase transition is not defined. For instance, the formation of $\alpha$ helices in proteins resembles crystallization. The nature of this helix-coil transition is a long-standing topic of research ${ }^{25}$ and has been studied with various methods including renormalization group techniques. ${ }^{26}$ A few years ago, additional evidence was presented by Hansmann and Okamoto ${ }^{27}$ and Alves and Hansmann ${ }^{28,29}$ that this helix-coil transition is for polyalanine (a homopolymer where in principle one can define the limes of an infinite chain), a true thermodynamic phase transition. Because of these previous results we decided to focus on helix formation in order to study the scaling behavior of short-time dynamics in proteins.

We define our order parameter as the number of helical residues $q_{H}=\max \left(\left(2<n_{H}(T)>/(N-2)-1\right), 0\right)$. Here we define a residue as helical if its backbone dihedral angles $(\phi, \psi)$ take values in the range $\left(-70^{\circ} \pm 30^{\circ},-37^{\circ} \pm 30^{\circ}\right.$ ) (Ref. 30) and the residue is hydrogen bonded. The normalization factor $N-2$ is chosen instead of $N$, the number of residues, because the flexible terminal residues are usually not part of an $\alpha$ helix. Our definition ensures that $0 \leqslant q_{H} \leqslant 1$ and $q_{h}\left(T_{c}\right)=0$.

Our short-time MC simulations of the helix-coil transition are based on a detailed, all-atom representation of proteins. The interaction between the atoms is described by a standard force field, empirical conformational energy program for peptides, version $2^{31}$ (ECEPP/2) as implemented in the program package SMMP (simple molecular mechanics for proteins), ${ }^{32}$

$$
\begin{aligned}
& E_{\mathrm{ECEPP} / 2}=E_{C}+E_{\mathrm{LJ}}+E_{\mathrm{HB}}+E_{\mathrm{tor}} \\
& E_{C}=\sum_{(i, j)} \frac{332 q_{i} q_{j}}{\varepsilon r_{i j}} \\
& E_{\mathrm{LJ}}=\sum_{(i, j)}\left(\frac{A_{i j}}{r_{i j}^{12}}-\frac{B_{i j}}{r_{i j}^{6}}\right)
\end{aligned}
$$

$$
\begin{aligned}
& E_{\mathrm{HB}}=\sum_{(i, j)}\left(\frac{C_{i j}}{r_{i j}^{12}}-\frac{D_{i j}}{r_{i j}^{10}}\right), \\
& E_{\mathrm{tor}}=\sum_{l} U_{l}\left(1 \pm \cos \left(n_{l} \chi_{l}\right)\right) .
\end{aligned}
$$

Here, $r_{i j}$ (in angstroms) is the distance between the atoms $i$ and $j$ and $\chi_{l}$ is the $l$ th torsion angle. The interactions between our polypeptides and water are approximated by means of an implicit water model which assumes that the solvation (free) energy is proportional to the solvent accessible surface area,

$$
E_{\mathrm{solv}}=\sum_{i} \sigma_{i} A_{i}
$$

where $E_{\mathrm{solv}}$ is the solvation energy, $A_{i}$ is the solvent accessible area of the $i$ th atom, and $\sigma_{i}$ is the corresponding solvation parameter. We have chosen the parameter set of Ref. 33 that is often used in conjunction with the ECEPP force field.

\section{RESULTS AND DISCUSSION}

We start our investigation of the short-time dynamics in the helix-coil transition by simulating polyalanine chains of lengths $N=10,20,30$, and 40 . Our results are averaged over 4000 independent runs for $N=10,20,30$, and 2000 runs for $N=40$. Errors are estimated by dividing these 4000 (2000) runs in bins of 100 (50) runs and calculating the fluctuation of the averages calculated for each bin. Figure 1(a) displays for various temperatures the time series of our order parameter $q_{h}$ as a function of Monte Carlo time for Ala 10 . Corresponding plots are displayed for $\mathrm{Ala}_{20}\left(\mathrm{Ala}_{30}\right)$ in Fig. 1(b) and 1(c), and for $\mathrm{Ala}_{40}$ in Fig. 1(d).

We expect to see in such log-log plot that curves of $q_{H}$ corresponding to low temperatures approach a constant value, while high temperatures are characterized by an exponential decrease of $n_{H}$. Both temperature regions are separated by the critical temperature $T_{c}$ where the corresponding curve is a straight line (in the scaling region). Our plots in Fig. 1 indicate for $N=10$ as critical temperature $T_{c}^{10}=315 \mathrm{~K}$, $T_{C}^{20}=415 \mathrm{~K}$ for $=20, T_{c}^{30}=450 \mathrm{~K}$ for $N=30$, and one of $T_{c}^{40}$ $=470 \mathrm{~K}$ for our longest chain, $\mathrm{Ala}_{40}$.

These estimates of the critical temperatures are lower than the ones presented in Ref. 34, where for $N=10,20$, and 30 the following temperatures were estimated from the position of the maximum in the specific heat: $T_{c}^{10}=333(2) \mathrm{K}$, $T_{c}^{20}=430(2) \mathrm{K}$, and $T_{c}^{30}=461(2) \mathrm{K}$. Hence, for these chain sizes the estimates obtained from a short-time analysis seem to be lower by $\approx 15 \mathrm{~K}$ than the ones estimated from the peak in specific heat. This does not indicate that one of the two sets is wrong. Even in spin systems the estimate of the critical temperature derived from the peak in the specific heat usually differs for small system size from the one obtained from the change in magnetization. Only for large system size both values converge. However, we remark that our values are calculated over a set of $4000(2000$ for $N=40)$ independent runs, while the results of multicanonical runs in Ref. 34 contain only of order $\approx 10$ independent events.

Note that the scaling relation leads to similar results for all chain lengths. This can be seen in Fig. 2 which displays 


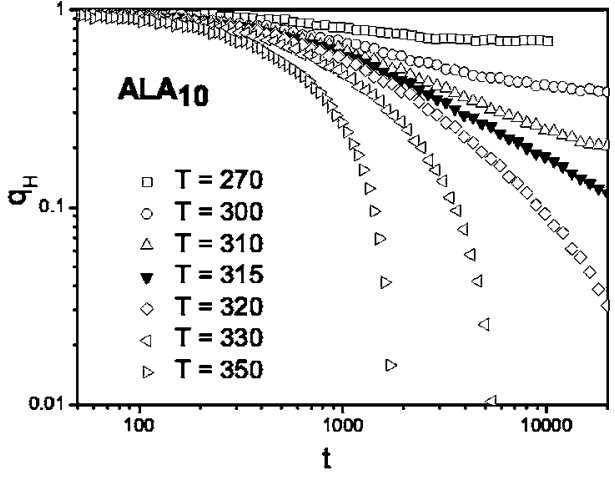

(a)

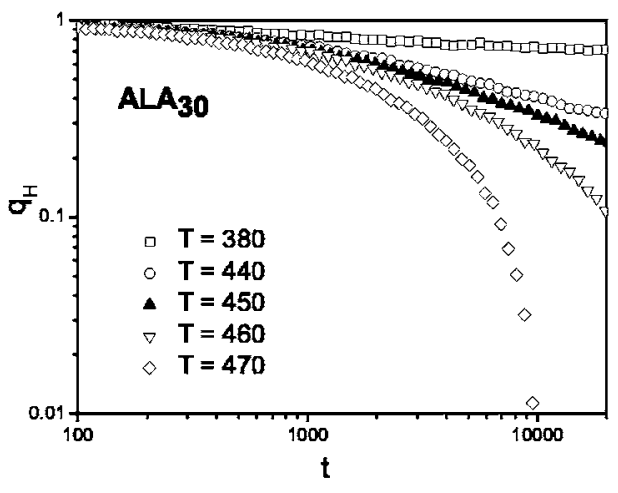

(b)

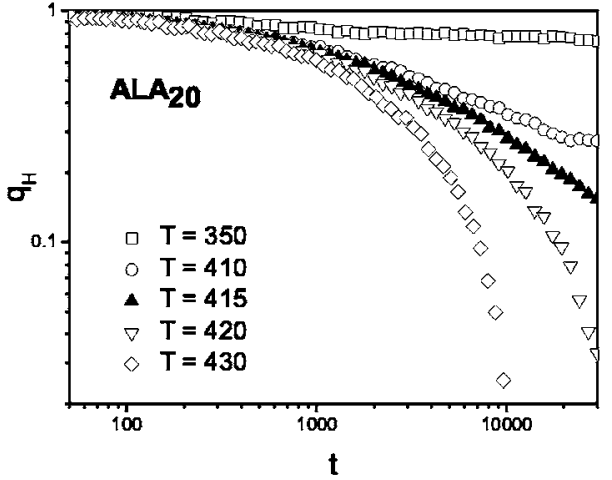

(c)

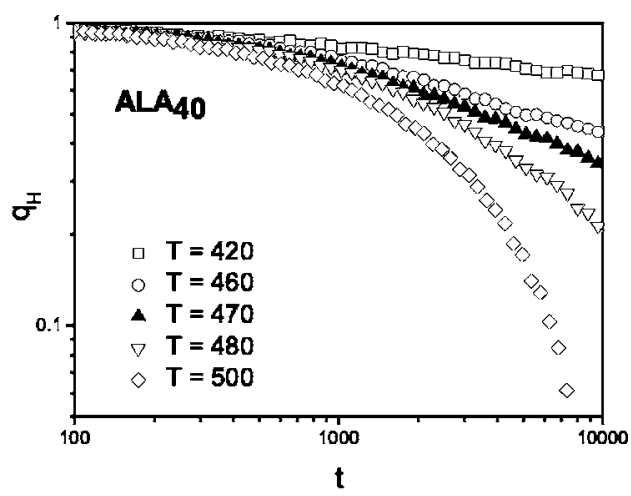

(d)
FIG. 1. Log-log plot of the time series of the helical order parameter $q_{H}$ (defined in the text) as a function of Monte Carlo time for polyalanine chains of length (a) $N=10$, (b) $N=20$, (c) $N=30$, and (d) $N=40$. for $T=T_{c}$ the time series of the order parameter as a function of time for all four chain lengths. We focus here on the linear range and show both the measured data points and the straight line that best fits these data. The data for the different chain sizes are almost parallel to each other and therefore differ little in their slope. From the slope of these plots we can obtain an estimate of $\beta / \nu z$.

Measuring in addition the slope for slightly higher (lower) temperatures and calculating the numerical derivative leads in addition to an estimate for $1 / \nu z$. In Fig. 3, the

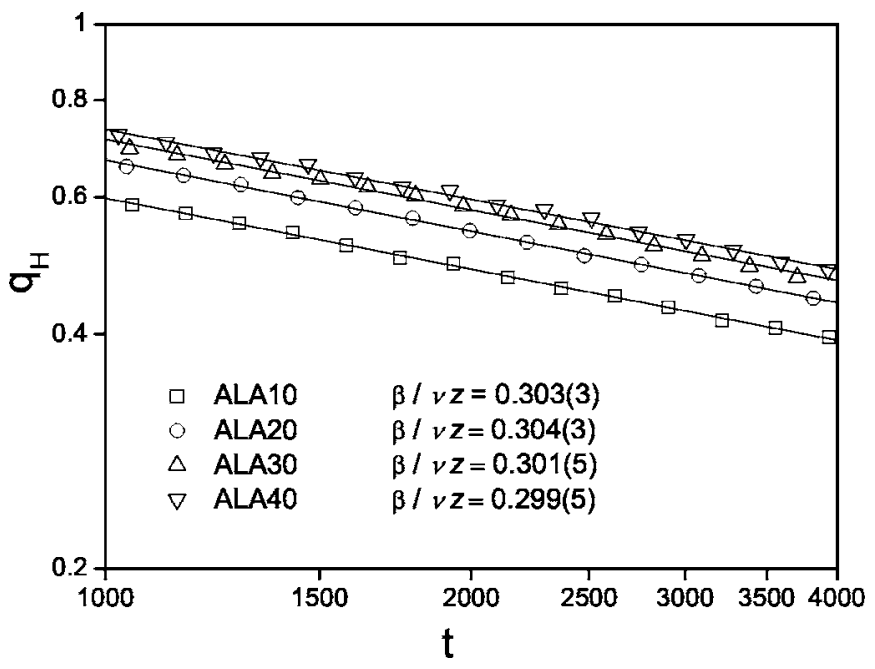

FIG. 2. Log-log plot of the time series of the helical order parameter $q_{H}$ as a function of Monte Carlo time for polyalanine chains of lengths $N=10,20$, 30 , and 40, as measured at their respective critical temperatures. Shown are the data in the scaling region and the best fit through them. power law increase of Eq. (2) is plotted in double-log scales for the for polyalanine chains. Estimates of the ratio $d / z$ can be extracted from the scaling of $U_{2}(t, L)$ which is shown for the four polyalanine chains in Fig. 4. Table I lists the values of all three ratios $\beta / \nu z, 1 / \nu z$, and $d / z$, together with the exponents $\beta$ and $d \nu$ that can be extracted from them.

In order to estimate the exponent $\theta_{g}$ one needs to prepare the initial states carefully to obtain precise values of the initial $q_{h}^{0}=q_{h}(t=0)$. The exponent $\theta_{g}$ is obtained from Eq. (5). Figure 5 shows the corresponding $\log -\log$ plots of $P(t)$ for $\mathrm{Ala}_{40}$ and various initial values of $q_{h}^{0}$. From these curves one

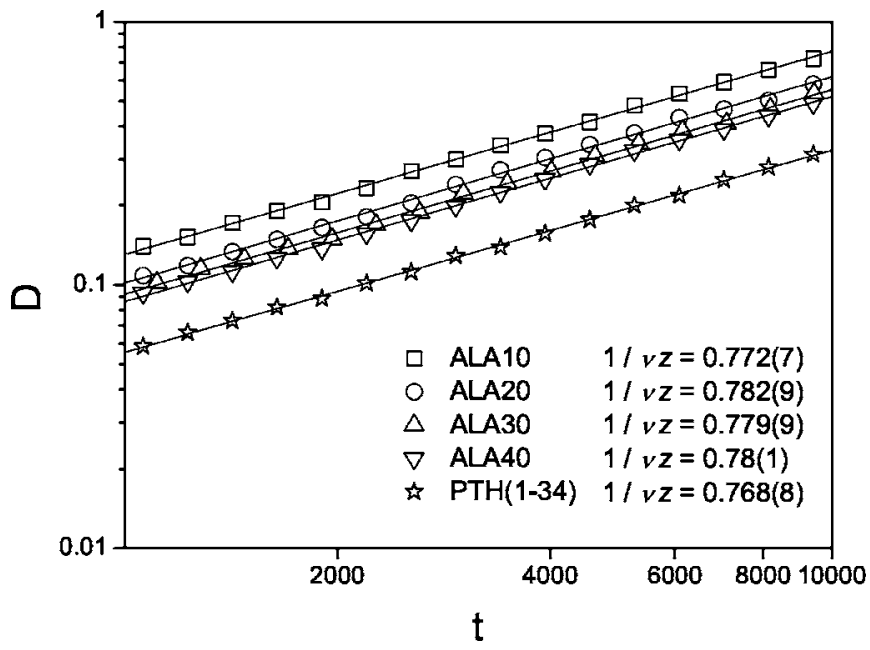

FIG. 3. Log-log plot of the time series of the logarithmic derivative of the order parameter [Eq. (2)] as a function of Monte Carlo time for polyalanine chains of lengths $N=10,20,30$, and 40, and for the polypeptide PTH(1-34). 


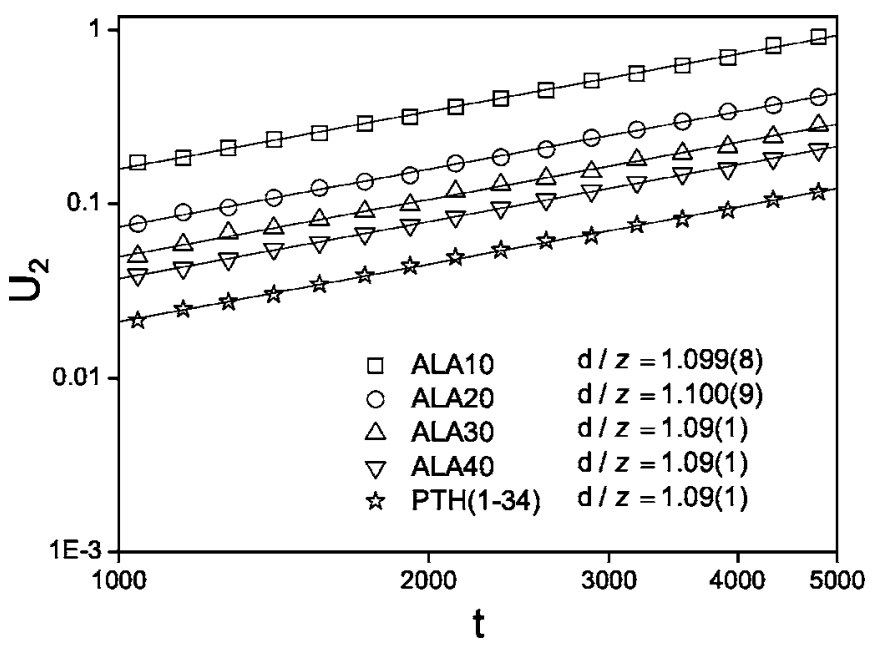

FIG. 4. Log-log plot of the time series of the second moment $F_{2}(t, L)$ as a function of Monte Carlo time for polyalanine chains of lengths $N=10,20$, 30 , and 40 and the polypeptide PTH(1-34), as measured at their respective critical temperatures. Shown are the data in the scaling region and the best fit through them.

extracts in the limes $N \rightarrow \infty$ and $q_{h}^{0} \rightarrow 0$ an estimate for the persistence exponent $\theta_{g}=0.73(1)$. The corresponding values for polyalanine chains of lengths $N=10,20,30$, and 40, and for the polypeptide PTH(1-34), are also listed in Table I.

Our values for the critical exponent $\beta \approx 0.39$ in Table I clearly exclude $\beta=0$, the value expected for a first order transition. A similar statement holds for $d \nu \approx 1.4$ that also excludes a first-order transition $(d \nu=1)$. This is different from the case of polyalanine in gas phase where the helixcoil transition is of first-order type. ${ }^{28}$

The estimates listed in Table I indicate that the finite size effects are less than the statistical errors and that reliable estimators for critical exponents can already be obtained on very small chains. This suggests that short-time analysis can also be applied to proteins where an extrapolation to infinite chain length is not possible. Here, short-time analysis opens a way to characterize thermodynamic processes in proteins by defining analog to phase transitions.

We have checked this assumption for the peptide fragment PTH(1-34) corresponding to residues 1-34 of human parathyroid hormone. ${ }^{35-37}$ This fragment is sufficient for the biological activities of the hormone suggesting medical and pharmaceutical applications of the peptide. The structure of PTH(1-34) has been resolved both crystallized ${ }^{36}$ and in solution $^{37}$ and is at room temperature almost completely helical. In previous work ${ }^{38}$ using the same energy function it

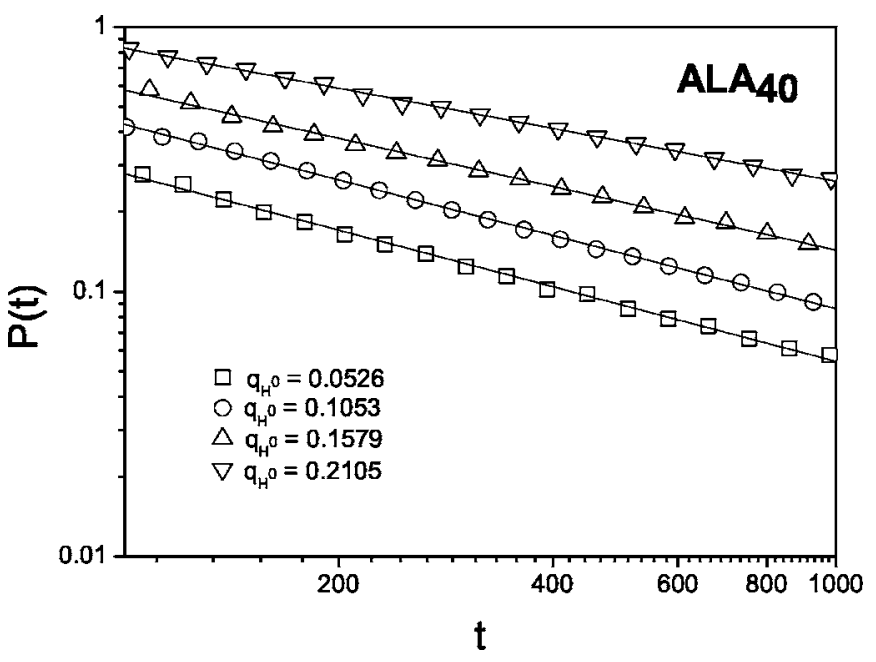

FIG. 5. Log-log plot of the time series of the global persistency $P(t)$ (defined in the text) for $\mathrm{Ala}_{40}$ and various initial order parameter values $q_{h}^{0}$.

was shown that PTH(1-34) exhibits at $T=560(10) \mathrm{K}$ a sharp transition between a high-temperature region where disordered coil structures prevail, and a low-temperature region that is characterized by mostly helical structures. The nature of this helix-coil transition could not be established in Ref. 38.

Observing the short-time dynamics of 500 runs for the helix-coil transition in $\mathrm{PTH}(1-34)$ we obtained data for temperatures $T=520,530,540,545,550$, and 560 K. Figure 6 displays our order parameter for various temperatures as a function of Monte Carlo time. From the plot we estimate that the critical temperature is $T=545(5) \mathrm{K}$, a value that is again slightly smaller than that obtained from the peak in specific heat. The scaling region is shown for this temperature in Fig. 7, with both the data point and the best straight-line fit through them.

Note that our estimate of the critical temperature differs slightly from the one $(T=540 \mathrm{~K})$ reported in Ref. 9. The slightly higher value that was obtained in this preliminary work with much lower statistics led to critical exponents different from the ones for polyalanine. Our new data indicate, however, that the critical exponents (listed also in Table I) are the same for the polyalanine chains and the polypeptide PTH(1-34). This is an interesting result as it indicates that the order of the helix-coil transition does not depend on the side chains of the residues in a protein. On one hand we have (poly)alanine with its extreme short side chain, on the other PTH(1-34) where the side chains differ for each

TABLE I. Exponents as obtained for polyalanine chains of length $N=10,20$, and 40 from the scaling relations of Eqs. (1)-(3) and (5). The columns 5 and 6 list the critical exponents $\beta$ and $d \nu$ as calculated from these quantities. The last row summarize the results for the polypeptide $\operatorname{PTH}(1-34)$.

\begin{tabular}{ccccccc}
\hline \hline$N$ & $\beta / \nu z$ & $1 / \nu z$ & $d / z$ & $\beta$ & $d \nu$ & $\theta_{g}$ \\
\hline 10 & $0.303(3)$ & $0.772(7)$ & $1.099(8)$ & $0.392(7)$ & $1.42(3)$ & $0.402(6)$ \\
20 & $0.304(3)$ & $0.782(9)$ & $1.100(9)$ & $0.389(8)$ & $1.40(3)$ & $0.687(7)$ \\
30 & $0.301(5)$ & $0.779(9)$ & $1.09(1)$ & $0.39(1)$ & $1.40(3)$ & $0.708(7)$ \\
40 & $0.299(5)$ & $0.78(1)$ & $1.09(1)$ & $0.38(1)$ & $1.42(4)$ & $0.710(8)$ \\
PTH & $0.290(5)$ & $0.768(8)$ & $1.09(1)$ & $0.38(1)$ & $1.42(3)$ & $0.705(6)$ \\
\hline \hline
\end{tabular}




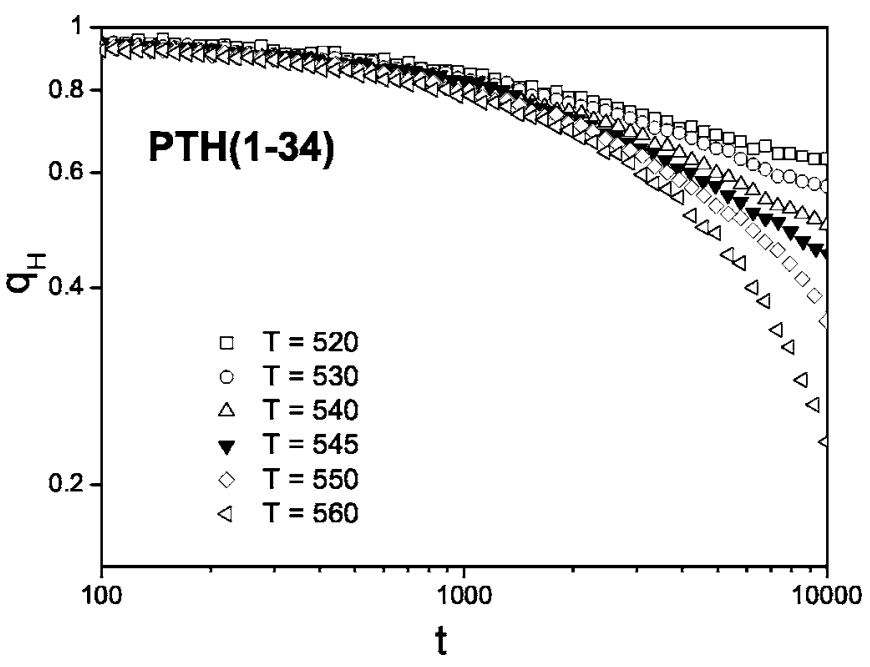

FIG. 6. Log-log plot of the time series of the helical order parameter $q_{H}$ as a function of Monte Carlo time for the polypeptide PTH(1-34) measured at temperatures $T=520,530,540,545,550$, and $560 \mathrm{~K}$.

residue and therefore side chain ordering is much more difficult to obtain than in a homopolymer. Hence, our new results suggest "universality" of the helix-coil transition in homopolymers and (helical) proteins.

\section{SUMMARY AND CONCLUSION}

We have investigated the short-time dynamics of helixforming polypeptides with up to $N=40$ residues. At their critical temperature the evolution of these molecules can be described by scaling laws that allow one to extract various critical exponents that characterize the helix-coil transition in these molecules. Our results indicate universality of the helix-coil transition in proteinlike molecules. They also demonstrate that the analysis of short-time dynamics is a

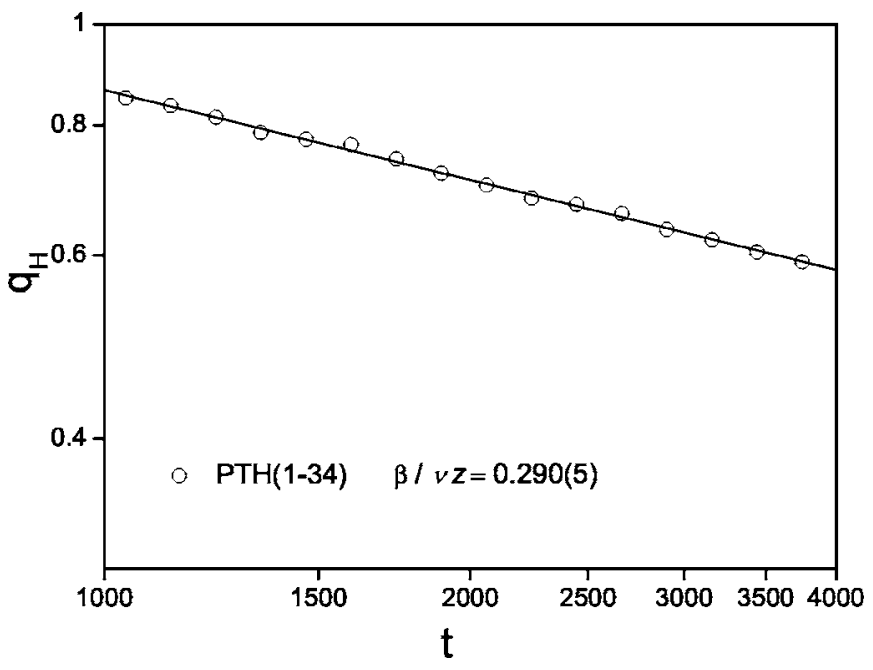

FIG. 7. Log-log plot of the time series of the helical order parameter $q_{H}$ as a function of Monte Carlo time for the polypeptide PTH(1-34) at the critical temperature $T_{c}=545$ in the scaling region, with the best fit also drawn through them. valuable tool for the investigation of transitions in proteins that may lead to a deeper understanding of their folding mechanism.

\section{ACKNOWLEDGMENTS}

Two of the authors (E.A. and J.R.D.F.) acknowledge support by Capes and CNPq (Brazil), and another author (U.H.E.H.) was supported by a research grant from the National Science Foundation (CHE-0313618).

${ }^{1}$ J. D. Bryngelson and P. G. Wolynes, Proc. Natl. Acad. Sci. U.S.A. 84, 7524 (1987)

${ }^{2}$ J. N. Onuchic, Z. Luhey-Schulten, and P. G. Wolynes, Annu. Rev. Phys. Chem. 48, 545 (1997).

${ }^{3}$ U. H. E. Hansmann, Comput. Sci. Eng. 5, 64 (2003).

${ }^{4}$ U. H. E. Hansmann and Y. Okamoto, Curr. Opin. Struct. Biol. 9, 177 (1999).

${ }^{5}$ U. H. E. Hansmann and Y. Okamoto, in Annual Reviews in Computational Physics VI, edited by D. Stauffer (World Scientific, Singapore, 1999), p. 129

${ }^{6}$ K. Hukushima and K. Nemoto, J. Phys. Soc. Jpn. 65, 1604 (1996); G. J. Geyer, Statist. Sci. 7, 437 (1992).

${ }^{7}$ P. M. C. de Oliveira, T. J. P. Penna, and H. J. Herrmann, Braz. J. Phys. 26, 677 (1996); P. M. C. de Oliveira, Int. J. Mod. Phys. C 9, 497 (1998). ${ }^{8}$ B. A. Berg and T. Neuhaus, Phys. Lett. B 267, 249 (1991).

${ }^{9}$ E. Arashiro, J. R. Drugowich de Felício, and U. H. E. Hansmann, Phys. Rev. E 73, 040902(R) (2006).

${ }^{10}$ H. K. Janssen, B. Schaub, and B. Schmittmann, Z. Phys. B: Condens. Matter 73, 539 (1989).

${ }^{11}$ D. A. Huse, Phys. Rev. B 40, 304 (1989).

${ }^{12}$ D. Stauffer, Physica A 186, 197 (1992).

${ }^{13}$ C. Münkel, D. W. Heermann, J. Adler, M. Gofman, and D. Stauffer, Physica A 193, 540 (1993).

${ }^{14}$ L. Schülke and B. Zheng, Phys. Lett. A 215, 81 (1996).

${ }^{15}$ Z. B. Li, L. Schülke, and B. Zheng, Phys. Rev. E 53, 2940 (1996).

${ }^{16}$ B. Zheng, Int. J. Mod. Phys. B 12, 1419 (1998).

${ }^{17}$ S. N. Majumdar, A. J. Bray, S. J. Cornell, and C. Sire, Phys. Rev. Lett. 77, 3704 (1996).

${ }^{18}$ S. N. Majumdar and A. J. Bray, Phys. Rev. Lett. 91, 030602 (2003).

${ }^{19}$ L. Schulke and B. Zheng, Phys. Lett. A 233, 93 (1997).

${ }^{20}$ H. Hinrichsen and H. M. Koduvely, Eur. Phys. J. B 5, 257 (1998).

${ }^{21}$ B. Zheng, Mod. Phys. Lett. B 16, 775 (2002).

${ }^{22}$ H. A. Fernandes, E. Arashiro, J. R. Drugowich de Felício, and A. A. Caparica, Physica A 366, 255 (2006).

${ }^{23}$ E. Arashiro, H. A. Fernandes, and J. R. Drugowich de Felício, e-print cond-mat/0603436.

${ }^{24}$ H. A. Fernandes and J. R. Drugowich de Felício, Phys. Rev. E 73, 057101 (2006).

${ }^{25}$ D. Poland and H. A. Scheraga, J. Chem. Phys. 45, 1456 (1966).

${ }^{26} \mathrm{Z}$. Li and H. A. Scheraga, J. Phys. Chem. 88, 6580 (1984).

${ }^{27}$ U. H. E. Hansmann and Y. Okamoto, J. Chem. Phys. 110, 1267 (1999); 111, 1339(E) (1999).

${ }^{28}$ N. A. Alves and U. H. E. Hansmann, Phys. Rev. Lett. 84, 1836 (2000).

${ }^{29}$ N. A. Alves and U. H. E. Hansmann, Physica A 292, 509 (2001).

${ }^{30}$ Y. Okamoto and U. H. E. Hansmann, J. Phys. Chem. 99, 11276 (1995).

${ }^{31}$ M. J. Sippl, G. Némethy, and H. A. Scheraga, J. Phys. Chem. 88, 6231 (1984), and references therein.

${ }^{32}$ F. Eisenmenger, U. H. E. Hansmann, Sh. Hayryan, and C.-K. Hu, Comput. Phys. Commun. 138, 192 (2001).

${ }^{33}$ T. Ooi, M. Obatake, G. Nemethy, and H. A. Scheraga, Proc. Natl. Acad. Sci. U.S.A. 8, 3086 (1987).

${ }^{34}$ Y. Peng, U. H. E. Hansmann, and N. A. Alves, J. Chem. Phys. 118, 2374 (2003).

${ }^{35}$ W. Klaus, T. Dieckmann, V. Wray, D. Schomburg, E. Wingender, and H. Mayer, Biochemistry 30, 6936 (1991).

${ }^{36}$ L. Jin, S. L. Briggs, S. Chandrasekhar, N. Y. Chirgadze, D. K. Clawson, R. W. Schevitz, D. L. Smiley, A. H. Tashjian, and F. Zhang, J. Biol. Chem. 275, 27238 (2000).

${ }^{37}$ U. C. Marx, K. Adermann, P. Bayer, W.-G. Forssmann, and P. Rösch, Biochem. Biophys. Res. Commun. 267, 213 (2000).

${ }^{38}$ U. H. E. Hansmann, J. Chem. Phys. 120, 417 (2004). 